\title{
Riqueza y diversidad de especies de aves asociadas a manglar en tres sistemas lagunares en la región costera de Oaxaca, México
}

\author{
Richness and diversity of bird species associated with mangrove in three lagoon systems in the \\ coastal region of Oaxaca, Mexico
}

\author{
José Cruz Bojorges-Baños \\ Instituto de Recursos, Universidad del Mar, Campus Puerto Escondido. Km. 2.1, Carretera a Sola de Vega, San 71980, Pedro Mixtepec, Oaxaca, \\ México. \\ Correspondencia: josebojorges@zicatela.umar.mx; josebojorges@hotmail.com
}

\begin{abstract}
Resumen. Se documentó la riqueza y diversidad avifaunística mediante capturas y conteos en las lagunas de Chacahua, Manialtepec y La Ventanilla. La diversidad de Shannon-Wiener se comparó mediante pruebas $t$ de Hutcheson y la similitud de la riqueza avifaunística y se determinaron diferencias entre la abundancia de especies. Se generaron curvas de acumulación de especies y se estimó la riqueza con el indicador de cobertura basado en frecuencia. Se registraron 17 órdenes, 39 familias y 94 especies: 69 residentes y 25 migratorias. La abundancia no presentó diferencias (p>0.05). Hubo diferencias en la diversidad de especies entre las lagunas; La Ventanilla presentó la más elevada (3.51). La mayor similitud se obtuvo entre Chacahua y Manialtepec con $56 \%$ y la menor entre Manialtepec y La Ventanilla con $42 \%$. La riqueza y proporción de especies residentes y migratorias fue similar en las 3 áreas; no obstante, por la baja similitud, se infiere que la diferencia en la composición de especies es significativa y que existe la posibilidad de registrar otras. Esta información complementa estudios previos, representa un inventario actualizado e indica que la avifauna asociada a manglar no se ha documentado totalmente.
\end{abstract}

Palabras clave: avifauna, diversidad, manglares, Oaxaca, riqueza.

\begin{abstract}
Avian richness and diversity was recorded in the lagoons of Chacahua, Manialtepec and La Ventanilla, using counts and captures. Shannon-Wiener diversity was compared using the Hutcheson's $t$-test, similarity of species richness was determined, as were differences in abundance. Species accumulation curves were generated and richness was estimated using the Incidence-based Estimator. 17 orders, 39 families and 94 species were registered, 65 of which were resident and 25 of which were migratory. While no difference in abundance was detected $(p>0.05)$, there was difference in diversity between the lagoons, with La Ventanilla presenting the highest index (3.51). The greatest similarity obtained was between Chacahua and Manialtepec, with 56\%, and the least was between Manialtepec and La Ventanilla, with $42 \%$. The richness and proportion of resident and migratory species was similar in the 3 areas; however, due to the low similarity, it is inferred that species composition differs significantly and that possibilities of registering other species exist. This information complements previous studies, it represents a current inventory and indicates that the avifauna associated with mangroves has not been totally documented.
\end{abstract}

Key words: avifauna, diversity, Oaxaca, mangrove, richness.

\section{Introducción}

La riqueza y diversidad de especies son propiedades emergentes de las comunidades biológicas y comúnmente son utilizadas para describir una taxocenosis, para determinar su distribución y presencia, para evaluar sus respuestas a las perturbaciones ambientales y para establecer planteamientos contemporáneos de conservación (Magurran, 1988; Gaston, 1996; Rosenstock et al., 2002). No obstante la importancia de estos atributos, existen pocas zonas de México con información que permita documentar la riqueza

Recibido: 17 agosto 2009; aceptado: 25 junio 2010 avifaunística, su abundancia, distribución y estacionalidad (Altamirano et al., 2002). Ejemplo de lo anterior son los manglares, los cuales, aún siendo hábitats importantes para la reproducción, refugio y alimentación de aves acuáticas y terrestres, migratorias y residentes (Mann, 1982; Naranjo, 1997) no han sido bien estudiados y el estado actual de sus poblaciones es relativamente desconocido.

Particularmente en la región costera de Oaxaca, pocos trabajos se han realizado en manglar con este grupo de vertebrados; la información que comprenden son inventarios básicos. Entre éstos destacan los estudios de la riqueza avifaunística en los sistemas donde se realizó el presente trabajo, los cuales mencionan el registro de 105 espe- 
cies para el Parque Nacional Lagunas de Chacahua, 268 para Manialtepec y 121 para La Ventanilla (Meléndez y Binnqüist, 1997; Meléndez, 2000; Arizmendi y MárquezValdelamar, 2000; Becerril-Morales, 2000; 2001).

Debido a que la biodiversidad sirve como base para explicar la estructura y función de las comunidades (Magurran, 1998; Watson y Peterson, 1999), el significado ecológico se incrementa al considerarse la información de la riqueza y abundancia de especies (Balmer, 2002); por lo tanto, es necesario cuantificar el tamaño de las poblaciones y delimitar las áreas preferidas por la avifauna residente y migratoria (Meléndez y Binnqüist, 1997). En el presente trabajo se estimó la riqueza y diversidad de especies de aves asociadas al manglar en 3 sistemas lagunares de la costa oaxaqueña y se actualizó el inventario de éstas.

\section{Materiales y métodos}

Este trabajo se realizó en la costa de Oaxaca (INEGI, 2000); las zonas de estudio fueron los bosques de manglares (Laguncularia racemosa y Rhizophora mangle) en los sistemas lagunares del Parque Nacional Lagunas de Chacahua $\left(15^{\circ} 58^{\prime} \mathrm{N}, 97^{\circ} 32^{\prime} \mathrm{O}\right)$, laguna de Manialtepec $\left(15^{\circ} 56^{\prime} \mathrm{N}, 97^{\circ} 10^{\prime} \mathrm{O}\right)$ y el estero La Ventanilla $\left(15^{\circ} 40^{\prime} \mathrm{N}\right.$, $\left.96^{\circ} 34^{\circ} \mathrm{O}\right)$. El muestreo de la avifauna se realizó en 6 sitios ubicados en el interior del bosque de mangle, 2 en cada zona de estudio, que se establecieron en función de la extensión del manglar y de la accesibilidad al mismo durante el muestreo. Las 3 zonas presentan inundación que varía en amplitud dependiendo de la temporada del año. Así, Chacahua y Manialtepec presentaron respectivamente franjas de mangle de 30 a $100 \mathrm{~m}$ de ancho con poca o nula inundación; mientras que en La Ventanilla los sitios de muestreo presentaron inundación permanente. Estas condiciones permitieron establecer sitios con superficies aproximadas de 7.5 ha en Chacahua y de 4 ha en Manialtepec y en La Ventanilla. Cada zona fue muestreada 2 días durante agosto, septiembre y octubre de 2006 y febrero de 2007. Durante noviembre y diciembre de 2006 y enero de 2007, la excesiva inundación de las lagunas La Ventanilla y Manialtepec imposibilitó los muestreos, debido a que la comunicación con el mar es efímera, por lo que la navegación es nula (Contreras y García-Nagaya, 1991; Becerril-Morales, 2001).

La captura de la avifauna se realizó con 12 redes ornitológicas colocadas dentro del bosque de mangle en sitios con separaciones de aproximadamente 100 metros, mediante muestreo no sistemático o recorridos aleatorios en las zonas de estudio, para realizar conteos y registrar todas las especies vistas y escuchadas (Fjeldså, 1999;
Bojorges y López-Mata, 2001; Bojorges y López-Mata, 2005: Bojorges et al., 2006). Ambas actividades se realizaron durante 8 horas a partir del amanecer. Las redes fueron revisadas aproximadamente cada 30 minutos, para evitar que los individuos capturados sufrieran daño. Cada ave capturada se marcó con un anillo de plástico colocado en el tarso y se liberó. Las especies de aves fueron identificadas visualmente mediante cotejo con las guías de campo de Howell y Webb (1995) y National Geographic (1999), y auditivamente con grabaciones obtenidas de Coffey y Coffey (1989).

La riqueza de especies fue la suma de las capturadas y las observadas no sistemáticamente. A cada especie de ave se le asignó un estatus de permanencia en el área, ya fuera residente o migratoria (Howell y Webb, 1995). La nomenclatura usada en este trabajo es la sugerida por la Unión de Ornitólogos Americanos (AOU, 2009); para los nombres en español se siguió a Escalante et al. (1996). La abundancia relativa de las especies se determinó con base en los siguientes porcentajes: abundante (90-100\%), común $(65-89 \%)$, moderadamente común (31-64\%), no común (10-30\%) y rara (1-9\%) (Pettingill, 1969), los que se obtuvieron al dividir el número de individuos de cada especie entre el número de individuos de todas las especies al término del muestreo, y el cociente se multiplicó por 100 para obtener el porcentaje de cada especie.

La similitud de la riqueza avifaunística entre las zonas de estudio se determinó con el coeficiente de comunidad de Jaccard, el cual es confiable para analizar datos de presencia-ausencia (Mueller-Dombois y Ellenberg, 1974). Diferencias significativas de la abundancia de especies entre las zonas se determinaron utilizando un análisis de Kruskal-Wallis (SAS Institute, 1999), que se realizó con las abundancias registradas de manera no sistemática en cada zona durante todo el muestreo.

Con los datos de riqueza y abundancia se estimó el índice de diversidad de Shannon-Wiener (H'), los valores obtenidos se compararon con pruebas $t$ de Hutcheson para detectar diferencias significativas entre éstos y determinar si un área es más diversa que otra (Zar, 1996).

Con base en la riqueza obtenida y con la abundancia de cada especie se estimó la riqueza posible en cada zona de estudio con el indicador de cobertura basado en frecuencia (IFC), incluido en el Programa EstimateS (Colwell, 2000). Para calcular el esfuerzo de muestreo necesario para registrar el mayor número de especies posibles en cada área de estudio se obtuvieron curvas de acumulación de especies. Es menester aclarar que para los análisis estadísticos aquí realizados se excluyeron las abundancias de las especies capturadas y sólo se consideraron especies vistas, tanto dentro del bosque de mangle como perchando en el dosel del mismo. Adicionalmente, se registraron las especies de 
aves que no utilizaron el bosque de mangle (i. e. perchando fuera del mangle) para tener pruebas de su presencia en las inmediaciones de las zonas de estudio. El listado obtenido se cotejó con las especies incluidas en la NOM-059-SEMARNAT- 2001 (SEMARNAT, 2002).

\section{Resultados}

Durante 192 horas de observación, se registraron 67 especies de aves en Chacahua, 72 en Manialtepec y 71 en La Ventanilla. El inventario obtenido para las 3 zonas de estudio fue de 94 especies: 69 residentes y 30 migratorias. De este total, 26 se observaron exclusivamente fuera del bosque de mangle mientras que 68 se ubicaron en el interior del mismo. La riqueza de especies está incluida en 17 órdenes y 39 familias (Apéndice 1). Chacahua compartió 31 especies con Manialtepec y 26 con La Ventanilla, la similitud entre estas áreas fue de 56 y $46 \%$ respectivamente. Manialtepec y La Ventanilla compartieron 25 especies y fueron similares en $42 \%$. Las especies comunes para las 3 áreas fueron 23: 16 residentes y 7 migratorias.

Un análisis comparativo de la riqueza de especies registrada (fuera y en el interior del bosque) en este trabajo y los inventarios previos realizados en estas localidades indican que 78, 77 y 66 especies ya se habían registrado en Chacahua, Manialtepec y en La Ventanilla, respectivamente. De las especies que se observaron exclusivamente en el interior del bosque, 29 se registraron anteriormente en Chacahua, 39 en Manialtepec y 29 en Ventanilla.

En el presente estudio se registraron especies que no se habían documentado previamente: 7 para Chacahua, 11 para Manialtepec y 18 para La Ventanilla. De las 3 áreas en conjunto,se agregaron 8 registros nuevos al inventario (Meléndez y Binnqüist, 1997; Meléndez, 2000; BecerrilMorales, 2000; 2001) : atila (Attila spadiceus), carpintero pico plata (Campephilus guatemalensis), cuclillo faisán (Dromococcyx phasianellus), chipe garganta amarilla (Dendroica dominica), papamoscas atigrado (Myiodynastes luteiventris), chipe peregrino (Vermivora peregrina), vireo manglero (Vireo pallens). De las 68 especies consideradas para los análisis de este trabajo, 44 son residentes y 24 migratorias. El total de individuos observados para Chacahua fue de 270, en Manialtepec se registraron 326 y en La Ventanilla 202. El número de aves capturadas en las 3 zonas de estudio fue de 394 y pertenecieron a 49 especies: 28 residentes y 21 migratorias. (Fig. 1B).

En Manialtepec se registraron 45 especies, 28 residentes y 17 migratorias; 5 especies [i.e. chipe charquero (Parkesia noveboracensis), chipe flameante (Setophaga ruticilla)] fueron abundantes, 20 escasas [i.e. colibrí canela
(Amazilia rutila), chivirín barrado (Thryothorus pleurostictus)] y 19 raras [i.e. zorzal de Swainson (Catharus ustulatus), tángara roja (Piranga rubra)]; en esta zona se capturaron 160 individuos de 30 especies: 15 residentes y 15 migratorias.

En Chacahua se registraron 42 especies, 31 residentes y 11 migratorias; 3 fueron abundantes, i.e. chipe charquero y chipe flameante, 10 escasas [i.e. mosquero californiano (Empidonax difficilis), parula norteña (Parula americana)] y 29 raras [i.e. rascador oliváceo (Arremonops rufivirgatus), tórtola coquita (Columbina passerina)]; en esta zona se capturaron 172 individuos de 24 especies: 14 residentes y 10 migratorias.

En La Ventanilla se observaron 40 especies, 28 residentes y 12 migratorias; 21 fueron escasas [i.e. colorín pecho naranja (Passerina leclancherii), bolsero de Altamira (Icterus gularis)] y 19 raras [i.e. paloma arroyera (Leptotila verreauxi), chipe dorado (Protonotaria citrea)]. No se registraron especies abundantes, se capturaron 60 individuos de 26 especies: 17 residentes y 9 migratorias (Fig. 1A).

La mayor abundancia para Chacahua y Manialtepec se registró en octubre de 2006, mientras que para La Ventanilla se obtuvo en febrero de 2007 (Fig. 2). La figura 3 muestra una comparación del número de individuos observados y capturados y el número de especies registradas por estos métodos. La abundancia de las especies registradas en las 3 áreas muestreadas no difirió significativamente $\left(\mathrm{H}_{2}\right.$ $=2.71, \mathrm{p}=0.25$ ).

De acuerdo con el estimador de diversidad (H'), La Ventanilla tuvo el valor más alto con 3.51, mientras que para Manialtepec y Chacahua se estimaron 3.29 y 2.98 respectivamente. La prueba de Hutcheson indicó que existieron diferencias significativas entre la diversidad de especies estimada para las 3 zonas: La Ventanilla y Chacahua $\left(\mathrm{t}_{384(2)}=5.99, \mathrm{p}<0.05\right)$, La Ventanilla y Manialtepec $\left(t_{521(2)}=-3.15, \mathrm{p}<0.05\right)$ y Chacahua y Manialtepec $\left(t_{499(2)}=\right.$ $3.26, \mathrm{p}<0.05$ ). Por otra parte, la riqueza de especies estimada para Manialtepec fue de casi 49, para Chacahua $47 \mathrm{y}$ alrededor de 42 para La Ventanilla . Las curvas de acumulación de especies mostraron un incremento creciente que no se estabilizó al final del muestreo (Fig. 3).

\section{Discusión}

El número de especies de aves registrado en los 3 sistemas lagunares representa el $33 \%$ de la riqueza anteriormente registrada para las mismas. Esto se debió principalmente a que los muestreos se limitaron al interior del bosque de mangle, lo que impidió el registro de numerosas especies 

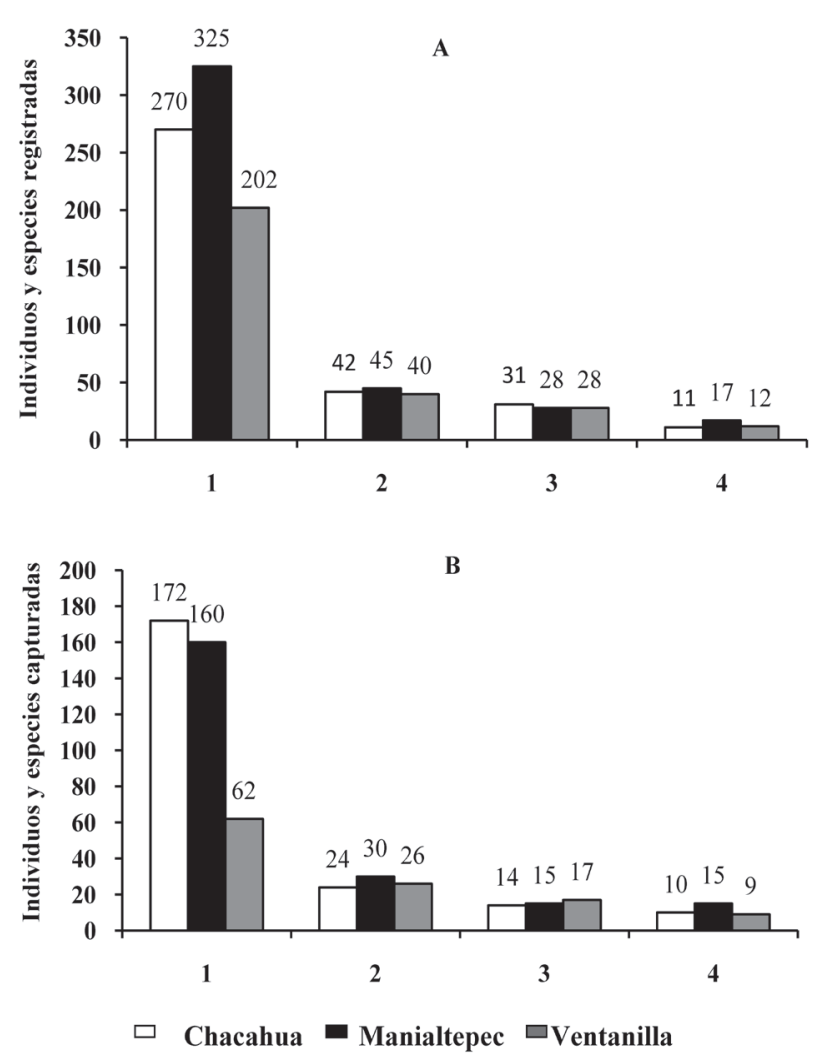

Figura 1. A. Abundancia de individuos observados y número de especies observadas. 1, individuos registrados; 2, especies registradas; 3, especies residentes; 4 , especies migratorias. B. Número de individuos capturados y número de especies capturadas. 1, individuos capturados; 2 , especies capturadas; 3 , especies residentes; 4, especies migratorias.

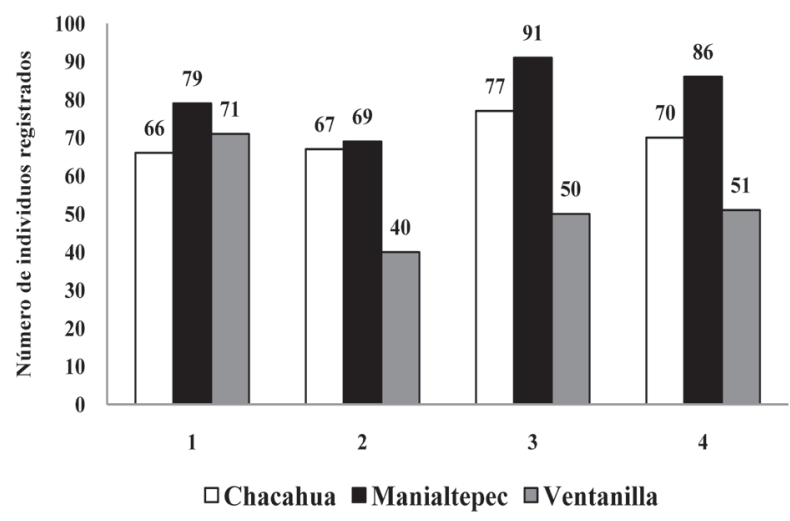

Figura 2. Número de individuos registrados en cada una de las lagunas durante el periodo de estudio. que pueden observarse en sus inmediaciones (i.e. garzas, playeros, rapaces, etc.) y que están bien documentadas en los inventarios previamente realizados; por ejemplo, en Manialtepec se han registrado hasta 24 especies de rapaces y 32 de aves playeras (Benítez et al., 1999).

La riqueza observada y la proporción de especies residentes y migratorias es similar en los 3 sistemas; sin embargo, comparten pocas especies, por lo que la similitud entre éstas es relativamente baja, sugiriendo diferencias en la composición de especies. Esta variación puede atribuirse a características físicas, como la superficie inundable de cada zona, la cual varía y expone superficies con poca o nula inundación, lo que es aprovechado por especies con distintos hábitos. En Chacahua, por ejemplo, se registraron más especies de palomas que en Manialtepec y La Ventanilla porque tienen hábitos terrestres y su presencia es menor en zonas inundadas. Similarmente, el chipe charquero (Parkesia noveboracensis) quien prefiere sitios poco inundados fue más abundante en Chacahua y Manialtepec. Asimismo, únicamente en La Ventanilla se registraron especies con hábitos acuáticos: pedrete coronaclara (Nyctanassa violacea) y garza cucharón (Cochlearius cochlearius), lo que sugiere que aun cuando se trate de un mismo tipo de hábitat pueden existir factores o características, como la superficie inundable o la estructura de la vegetación, que expliquen la presencia de algunas especies y la ausencia de otras. Esto pudiera ser apoyado por los inventarios previos, en los cuales comparativamente se observan diferencias en la riqueza de especies para cada laguna.

Además de lo anterior, la cantidad de especies abundantes, escasas y raras registradas en cada zona de estudio, le confiere variabilidad a la diversidad, lo que es apoyado con las diferencias estadísticas obtenidas con la prueba de Hutcheson. Así, aun cuando en La Ventanilla se registró la menor riqueza de especies, ninguna fue abundante y la proporción de las raras y escasas fue similar, influyendo en el valor de diversidad estimado, que será mayor si no hay especies dominantes. Por otra parte, en Chacahua y Manialtepec sí se registraron especies abundantes, mismas que aunadas a las raras le confirieron valores bajos de diversidad. Por lo tanto, debido al interés de mantener la máxima diversidad de especies de aves, la conservación del sistema de manglares en esta región será necesaria. La información sobre la abundancia de las especies registradas es importante, ya que son datos que le confieren mayor significado ecológico a la estructura comunitaria (Balmer, 2002) al reconocer, por ejemplo, especies dominantes. Un análisis fino de la dinámica comunitaria así como de la estratificación de las especies de la avifauna sería necesario para explicar la proporción de especies abundantes, escasas y raras en cada área de estudio. 
A

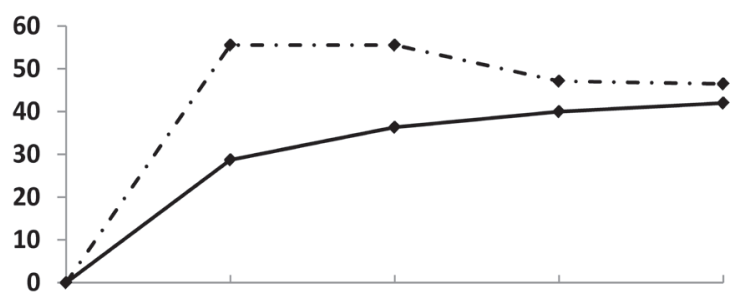

B

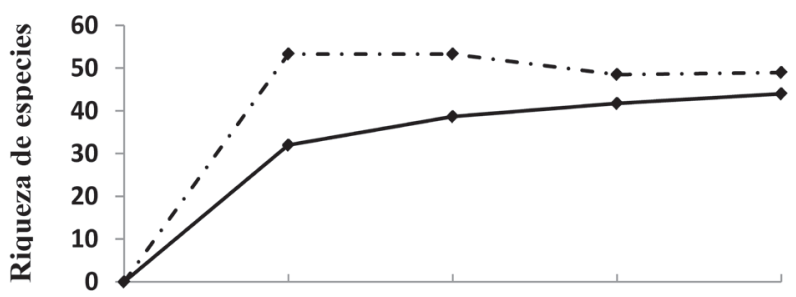

C

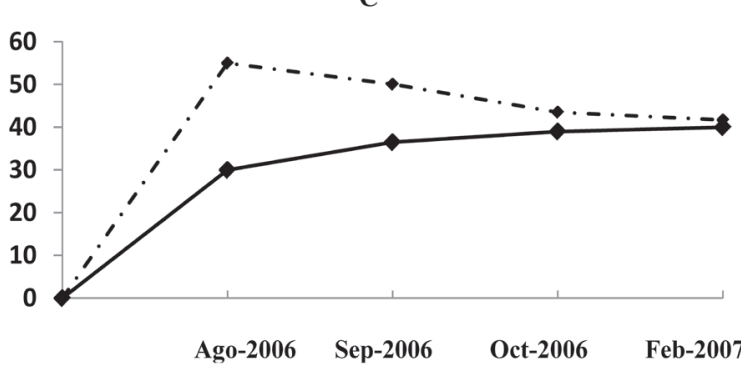

Figura 3. Curvas de acumulación de especies. A, especies observadas y estimadas en Chacahua; B, especies observadas y estimadas en Manialtepec; C, especies observadas y estimadas en La Ventanilla. Línea continua, especies observadas; línea discontinua, especies estimadas.

De acuerdo con la información disponible en los trabajos relacionados con las zonas de estudio, en Manialtepec se ha registrado el mayor número de especies de aves, sobrepasando por mucho la riqueza observada en Chacahua y La Ventanilla. No obstante, los resultados obtenidos en el presente trabajo indican que la avifauna asociada a manglar aún no ha sido totalmente documentada, y que el esfuerzo de muestreo debe incrementarse, realizando observaciones de la avifauna dentro del bosque de mangle. En este sentido, la documentación de la riqueza de especies de aves de una región debe contemplar muestreos en diferentes temporadas y ambientes para tener éxito en la realización del inventario y lograr el registro de la mayor cantidad de especies posible.

Las curvas de acumulación de especies sugieren la posibilidad de adicionar especies en inventarios subse- cuentes; la riqueza observada en cada una de las zonas representa el 95,88 y $90 \%$ de la riqueza estimada para $\mathrm{La}$ Ventanilla, Chacahua y Manialtepec, respectivamente.

La carencia de estudios similares en ambientes semejantes dificulta la comparación de los resultados aquí presentados, limitando la deducción sobre la riqueza y la abundancia de especies de aves en el interior del bosque de mangle. No obstante, aun cuando la riqueza de especies obtenida en este trabajo pudiera considerarse relativamente baja, la adición de otras especies a los inventarios previos de los 3 sistemas lagunares y a cada uno de éstos en particular, le confiere gran importancia. Esta información además de complementar las investigaciones previas realizadas por Meléndez y Binnqüist (1997), Benítez et al. (1999), Meléndez (2000), Becerril-Morales (2000, 2001) presenta un inventario que incrementa el conocimiento sobre la avifauna que regularmente se puede encontrar en estos hábitats.

En otro sentido, únicamente se recapturaron 5 individuos; 3 fueron migratorios: chipe charquero (Parkesia noveboracensis), chipe flameante (Parkesia ruticilla) y chipe trepador (Mniotilta varia), y 2 residentes: chivirín barrado (T. pleurostictus) y el martínpescador verde (Chloroceryle americana). Las recapturas se realizaron en diferentes fechas, pero en las mismas áreas donde se capturaron. Esta información limita el aseverar que la avifauna registrada en este estudio utiliza los manglares como corredor biológico. No obstante, la relativa cercanía de estos sistemas, las especies que comparten y la similitud de ambientes, permite suponer que la avifauna utiliza estas zonas como sitios de anidación, alimentación, refugio y descanso, como ha sido señalado por varios autores. Estos sitios, sobre todo para especies visitantes, representarían islas donde las aves podrían acumular reservas energéticas para una migración exitosa.

El estudio de la distribución de la diversidad es un tema central en ecología; determinar los procesos que inciden en los patrones de la riqueza de especies y si éstos actúan de manera similar sobre todas las especies y en diferentes escalas, puede responder cuestionamientos sobre la estructura y función de las comunidades (Watson y Peterson, 1999). En este sentido, el monitoreo continuo de la avifauna presente en este sistema de manglares, permitirá inferir sobre su sensibilidad a cambios ambientales, al identificar causas y efectos espaciales y temporales. Adicionalmente, podrá razonarse sobre la dinámica poblacional de especies individuales y comprender la variación de la diversidad.

La metodología utilizada permitió documentar la diversidad biológica de manera relativamente sencilla y rápida; así, mediante recorridos aleatorios se registró el $90.4 \%$ de la riqueza, mientras que con las capturas se obtuvo en cada 
laguna más del $50 \%$ de la riqueza avifaunística, la cual también fue observada; no obstante, únicamente por este medio se registraron 9 especies, lo que representa el 9.6\% de la riqueza total. Dicha metodología puede ser utilizada como referencia para registrar mediante el monitoreo cambios espaciales y temporales de la diversidad y composición de especies asociados a distintos factores, principalmente a los antropogénicos (Bojorges y López-Mata, 2005). En este sentido, las acciones para dirigir esfuerzos de conservación en estos sistemas deben incluir el monitoreo continuo de las poblaciones de aves, para detectar especies susceptibles a las modificaciones del ambiente. Adicionalmente, se deberán registrar variaciones en la distribución, riqueza y diversidad de la avifauna e identificar elementos asociados a estas variaciones, como amenazas comunes a la conservación de las poblaciones de aves; por ejemplo, la apertura de nuevos sitios de cultivo, ganadería, etc. Tales actividades fueron evidentes principalmente en Chacahua y Manialtepec, donde la primera, a pesar de ser una área natural protegida, exhibió deforestación y ganadería en índices no cuantificables, en tanto que para la segunda, la deforestación fue notoría en este estudio.

\section{Agradecimientos}

El presente trabajo fue financiado por la Universidad del Mar. En el trabajo de campo colaboraron A. Buenrostro Silva, J. García Grajales, M. A. Medina Carballido G. Ramos Olivera, J. A. Pérez Carbajal y M. Zúñiga Acevedo. El Parque Nacional Lagunas de Chacahua, la agencia del pueblo de Manialtepec y la cooperativa de La Ventanilla proporcionaron las facilidades necesarias para la realización de este estudio. Los comentarios de revisores anónimos mejoraron sustancialmente el contenido del manuscrito.

\section{Literatura citada}

Altamirano González-Ortega, M. A., M. F. Martin y G. J. Cartas. 2002. Ocurrencia, distribución y abundancia del género Passerina en la Reserva de la Biosfera La Sepultura, Chiapas. Acta Zoológica Mexicana (n.s.) 75:125-142.

AOU (American Ornithologists' Union). 2009. Check-list of North American birds. http://www.aou.org/checklist/north/ full.php; última consulta: 5.V.2009.

Arizmendi, M. C. y L. Márquez-Valdelamar. 2000. Áreas de importancia para la conservación de las aves en México. Consejo Internacional para la Preservación de las avesMéxico. Fondo Mexicano para la Conservación de la
Naturaleza. México, D.F. 440 p.

Balmer, O. 2002. Species list in ecology and conservation: abundances matter. Conservation Biology 16:1160-1161.

Becerril-Morales, F. 2000. Avifauna de dos sistemas lagunares costeros: Chacahua-Pastoría y Corralero-Alotengo, Oaxaca, 1998-1999. Resúmenes. XII Congreso Nacional de Oceanografía. 22 al 26 de mayo, Huatulco, Oaxaca, México. Becerril-Morales, F. 2001. Algunos aspectos sobre la ecología y conservación en el estero La Ventanilla, Oaxaca, México. Ciencia y Mar 15:37-45.

Benítez, H., C. Arizmendi y L. Márquez-Valdelamar. 1999. Base de datos de las AICAS. Consejo Internacional para la Preservación de las aves-México / Comisión Nacional para el Conocimiento y Uso de la Biodiversidad / Fondo Mexicano par la Conservación de la Naturaleza /CCA-México, D.F. (http://conabioweb.conabio.gob.mx/aicas_progs/buscar. pl?aica=221); última consulta: 5.V.2009

Bojorges, B. J. C. y L. López-Mata. 2001. Abundancia y distribución temporal de aves en una selva mediana subperennifolia en el centro de Veracruz, México. Anales del Instituto de Biología. Universidad Nacional Autónoma de México. Serie Zoología 72:259-283.

Bojorges B.J.C. y L. López-Mata. 2005. Riqueza y diversidad de especies de aves en una selva mediana subperennifolia del centro de Veracruz, México. Acta Zoológica Mexicana (n.s.) 21: 1-20.

Bojorges B. J. C., L. López-Mata, A. L. Tarango, H. J. G. Herrera y M. G. D. Mendoza. 2006. Combinación de métodos de muestreo para registrar la riqueza de especies de aves en ambientes tropicales. Universidad y Ciencia 22:111-118.

Coffey, B. B. y L. C. Coffey. 1989. Songs of Mexican birds. Cassette. ARA Records, Gainesville, Florida.

Colwell, R. K. 2000. EstimateS: statistical estimation of species richness and shared species from samples. Version 6.0. http:// viceroy.eeb.uconn.edu/estimates; última consulta: 5.V.2009.

Escalante, P., A. M. Sada y G. J. Robles 1996. Listado de nombres comunes de las aves de México. Comisión Nacional para el Conocimiento y Uso de la Biodiversidad, México, D.F. 32 p.

Contreras, F. y A, García-Nagaya. 1991. Hidrología, nutrientes y productividad primaria en la laguna San José Manialtepec Oaxaca, México. Hidrobiológica 1:65-72.

Fjeldså, J. 1999. The impact of human forest disturbance on the endemic avifauna of the Udzungwa Mountains Tanzania. Bird Conservation International 9:47-62.

Gaston, K. J. 1996. Species richness: measure and measurement. In Biodiversity: a biology by numbers and difference, K. J. Gaston (ed.). Blackwell Science, Oxford. p. 77-113.

Howell, S. N. G. y S. Webb 1995. A guide to the birds of Mexico and northern Central America. Oxford University Press, New York. $851 \mathrm{p}$.

INEGI (Instituto Nacional de Estadística, Geografía e Informática). 2000. Anuario estadístico del Estado de Oaxaca. 
Marco Geoestadístico. Aguascalientes, Aguascalientes.

Magurran, A. E. 1988. Ecological diversity and its measurement. Princeton University Press, New Jersey. 179 p.

Mann, K. 1982. Ecology of coastal waters: a system approach. University of California, Berkeley. 322 p.

Meléndez, A. y G. S. Binnqüist. 1997. Avifauna de los humedales costeros de Chiapas y Oaxaca. Ciencia y Mar 1:45-52.

Meléndez, H. A. 2000. AICA 221 Laguna de Manialtepec. In Áreas de Importancia para la Conservación de las Aves en México, M. C. Arizmendi y L. Márquez (eds.). Consejo Internacional para la Preservación de las aves. México, D.F. p. 201.

Muller-Dombois, D. y H. Ellenberg. 1974. Aims and methods of vegetation ecology. John Wiley, New York. 574 p.

Naranjo, L. G. 1997. A note on the birds of the Pacific mangroves of Colombia. In Mangrove ecosystem studies in Latin America and Africa, B. Kjerfve, L. D. de La Cerda y H. S. Diop (eds.). UNESCO-International Society for Mangrove Ecosystems. Forest Service Department of Agriculture, Paris. p. 64-70.

National Geographic. 1999. Field guide to the birds of North
America, third edition. National Geographic Society, Washington, D.C.:480 p.

Pettingill, O. S. Jr. 1969. Ornithology in laboratory and field. Burgess, Minneapolis, Minnesota.

Rosenstock, S. S., D. R. Anderson, K. M. Giesen, T. Leukering y M. F. Carter. 2002. Landbird counting techniques: Current practices and an alternative. Auk 119:46-53.

Watson, D. M. y A. T. Peterson. 1999. Determinants of diversity in a naturally fragmented landscape: bird faunas of humid montane forests in Mesoamerica. Ecography 22:582-589.

SAS Institute. 1999. SAS/STAT User's Guide, version 8. Cary, North Carolina.

SEMARNAT (Secretaría del Medio Ambiente y Recursos Naturales). 2002. Norma Oficial Mexicana NOM-059SEMARNAT-2001. Protección ambiental-especies nativas de México de flora y fauna silvestres-categorías de riesgo y especificaciones para su inclusión, exclusión o cambio-lista de especies en riesgo. Diario Oficial de la Federación, 6 de marzo.

Zar, J. H. 1996. Biostatistical analysis. Prentice Hall, New Jersey. $662 \mathrm{p}$.

Apéndice 1. Avifauna registrada en la zona de estudio. Estacionalidad, R: residente, M: migratoria. NOM-059, Pr: Protección especial, A: Amenazada. Se presentan los individuos registrados en cada laguna, In: registrado en el interior del bosque de mangle, Cp: capturada, Ex: registrada fuera del bosque de mangle. Abundancia relativa, R: rara, NC: no común, MC: moderadamente común, C: común y A: abundante. El asterisco después del nombre científico de las especies señala que éstas se utilizaron para los análisis estadísticos.

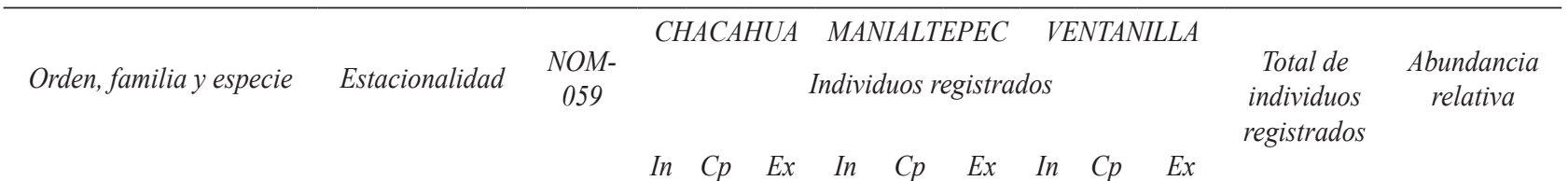

\section{ANSERIFORMES}

ANATIDAE

Anas discors *

M

1

1

$\mathrm{R}$

GALLIFORMES

CRACIDAE

Ortalis poliocephala

$\mathrm{R}$

6

4

5

15

NC

ODONTOPHORIDAE

Colinus virginianus

$\mathrm{R}$

23

23

$\mathrm{NC}$

PELECANIFORMES

PELECANIDAE

Pelecanus occidentalis

M

15

15

$\mathrm{NC}$ 
Apéndice 1. Continúa.

\begin{tabular}{|c|c|c|c|c|c|c|c|c|c|c|c|c|c|}
\hline \multirow[t]{2}{*}{ Orden, familia y especie } & \multirow[t]{2}{*}{ Estacionalidad } & \multirow[t]{2}{*}{$\begin{array}{c}\text { NOM- } \\
059\end{array}$} & \multicolumn{8}{|c|}{ Individuos registrados } & & \multirow{2}{*}{$\begin{array}{l}\text { Total de } \\
\text { individuos } \\
\text { registrados }\end{array}$} & \multirow[t]{2}{*}{$\begin{array}{l}\text { Abundancic } \\
\text { relativa }\end{array}$} \\
\hline & & & In & $C p$ & $E x$ & In & $C p$ & $E x$ & In & $C p$ & $E x$ & & \\
\hline Anhinga anhinga $*$ & $\mathrm{R}$ & & 2 & & & 6 & & & 5 & & & 13 & $\mathrm{NC}$ \\
\hline FREGATIDAE & & & & & & & & & & & & & \\
\hline Fregata magnificens & M & & & & 1 & & & 2 & & & 4 & 7 & $\mathrm{R}$ \\
\hline CICONIIFORMES & & & & & & & & & & & & & \\
\hline ARDEIDAE & & & & & & & & & & & & & \\
\hline Tigrisoma mexicanum * & $\mathrm{R}$ & $\operatorname{Pr}$ & 1 & & & & & & & & & 1 & $\mathrm{R}$ \\
\hline Ardea alba* & $\mathrm{R}$ & & 1 & & & 3 & & & 1 & & & 5 & $\mathrm{R}$ \\
\hline Egretta thula * & $\mathrm{R}$ & & 2 & & & 5 & & & 3 & & & 10 & $\mathrm{R}$ \\
\hline Bubulcus ibis * & $\mathrm{R}$ & & & & 3 & & & & 4 & & & 7 & $\mathrm{R}$ \\
\hline Butorides virescens * & $\mathrm{R}$ & & 5 & & & 3 & & & 10 & 5 & & 23 & $\mathrm{NC}$ \\
\hline Nyctanassa violacea $*$ & $\mathrm{R}$ & A & & & & & & & 2 & & & 2 & $\mathrm{R}$ \\
\hline Cochlearius cochlearius * & $\mathrm{R}$ & & & & & & & & 3 & & & 3 & $\mathrm{R}$ \\
\hline FALCONIFORMES & & & & & & & & & & & & & \\
\hline CATHARTIDAE & & & & & & & & & & & & & \\
\hline Coragyps atratus & $\mathrm{R}$ & & & & 3 & & & 7 & & & 4 & 14 & $\mathrm{NC}$ \\
\hline Cathartes aura & $\mathrm{R}$ & & & & 7 & & & 2 & & & 1 & 10 & $\mathrm{R}$ \\
\hline ACCIPITRIDAE & & & & & & & & & & & & & \\
\hline Pandion haliaetus & M & & & & 2 & & & 1 & & & 1 & 4 & $\mathrm{R}$ \\
\hline Buteogallus anthracinus & $\mathrm{R}$ & $\operatorname{Pr}$ & & & 1 & & & 1 & & & 1 & 3 & $\mathrm{R}$ \\
\hline Buteo magnirostris * & $\mathrm{R}$ & & & & 2 & 3 & 1 & & & & 2 & 7 & $\mathrm{R}$ \\
\hline Buteo nitidus & $\mathrm{R}$ & & & & 3 & & & 2 & & & 1 & 6 & $\mathrm{R}$ \\
\hline FALCONIDAE & & & & & & & & & & & & & \\
\hline Caracara cheriway & $\mathrm{R}$ & & & & 1 & & & 2 & & & 2 & 5 & $\mathrm{R}$ \\
\hline CHARADRIIFORMES & & & & & & & & & & & & & \\
\hline SCOLOPACIDAE & & & & & & & & & & & & & \\
\hline Actitis macularius $*$ & M & & 2 & & & 5 & & & 3 & & & 10 & $\mathrm{R}$ \\
\hline COLUMBIFORMES & & & & & & & & & & & & & \\
\hline COLUMBIDAE & & & & & & & & & & & & & \\
\hline Patagioenas flavirostris & $\mathrm{R}$ & & & & 3 & & & 7 & & & 7 & 17 & $\mathrm{NC}$ \\
\hline Zenaida asiatica $*$ & $\mathrm{R}$ & & 5 & & & 7 & 1 & & & & 3 & 15 & $\mathrm{NC}$ \\
\hline Columbina inca $*$ & $\mathrm{R}$ & & 3 & 6 & & 9 & 5 & & 9 & 1 & & 21 & $\mathrm{NC}$ \\
\hline Columbina passerina $*$ & $\mathrm{R}$ & & 1 & 1 & & 3 & 2 & & & & & 4 & $\mathrm{R}$ \\
\hline Columbina talpacoti $*$ & $\mathrm{R}$ & & 2 & 1 & & & & 5 & & & & 6 & $\mathrm{R}$ \\
\hline Leptotila verreauxi $*$ & $\mathrm{R}$ & & 5 & 2 & & & & 2 & 2 & 1 & & 9 & $\mathrm{R}$ \\
\hline PSITTACIFORMES & & & & & & & & & & & & & \\
\hline PSITTACIDAE & & & & & & & & & & & & & \\
\hline Aratinga canicularis * & $\mathrm{R}$ & $\operatorname{Pr}$ & 8 & & & & & 6 & & & 3 & 17 & $\mathrm{NC}$ \\
\hline Amazona albifrons * & $\mathrm{R}$ & & 2 & & & & & & & & & 2 & $\mathrm{R}$ \\
\hline
\end{tabular}


Apéndice 1. Continúa.

Orden, familia y especie

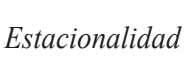

NOM
059

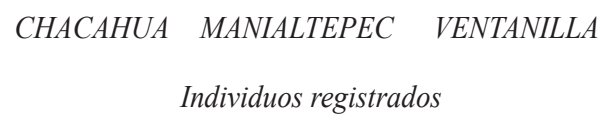

In $\quad C p \quad E x \quad$ In $\quad C p \quad E x \quad$ In $\quad C p \quad E x$

\section{CUCULIFORMES}

\section{CUCULIDAE}

Piaya cayana

Dromococcyx phasianellus

*

Morococcyx erythropygus

Crotophaga sulcirostris

STRIGIFORMES

\section{STRIGIDAE}

Glaucidium brasilianum

$\mathrm{R}$

Ciccaba virgata

$\mathrm{R}$

$\mathrm{R}$

$\mathrm{R}$

$\mathrm{R}$

CAPRIMULGIFORMES

\section{CAPRIMULGIDAE}

Nyctidromus albicollis

$\mathrm{R}$

APODIFORMES

TROCHILIDAE

Amazilia rutila *

TROGONIFORMES

\section{TROGONIDAE}

Trogon citreolus *

CORACIIFORMES

MOMOTIDAE

Momotus mexicanus

$\mathrm{R}$

ALCEDINIDAE

Megaceryle torquata*

Megaceryle alcyon *

Chloroceryle americana * PICIFORMES

PICIDAE

Melanerpes chrysogenys *

$\mathrm{R}$

Dryocopus lineatus *

Campephilus

guatemalensis

PASSERIFORMES

FURNARIIDAE

Xiphorhynchus flavigaster

TYRANNIDAE

Empidonax difficilis *

Attila spadiceus *
Total de individuos registrados

Abundancia relativa 


\section{Apéndice 1. Continúa.}

\begin{tabular}{|c|c|c|c|c|c|c|c|c|c|c|c|c|c|}
\hline \multirow[t]{2}{*}{ Orden, familia y especie } & \multirow[t]{2}{*}{ Estacionalidad } & \multirow[t]{2}{*}{$\begin{array}{c}\text { NOM- } \\
059\end{array}$} & \multicolumn{3}{|c|}{ CHACAHUA } & \multicolumn{3}{|c|}{ MANIALTEPEC } & \multicolumn{3}{|c|}{ VENTANILLA } & \multirow[t]{2}{*}{$\begin{array}{l}\text { Total de } \\
\text { individuos } \\
\text { registrados }\end{array}$} & \multirow[t]{2}{*}{$\begin{array}{c}\text { Abundancia } \\
\text { relativa }\end{array}$} \\
\hline & & & In & $C p$ & $E x$ & In & $C p$ & $E x$ & In & $C p$ & $E x$ & & \\
\hline Myiarchus tyrannulus * & $\mathrm{R}$ & & 3 & 4 & & & & 4 & & & & 7 & $\mathrm{R}$ \\
\hline Pitangus sulphuratus * & $\mathrm{R}$ & & 2 & 3 & & 14 & 17 & & 5 & 1 & & 21 & $\mathrm{NC}$ \\
\hline Myiozetetes similis * & $\mathrm{R}$ & & 1 & & & 10 & & & 8 & & & 19 & $\mathrm{NC}$ \\
\hline Myiodynastes luteiventris & M & & & & 3 & & & 6 & & & 4 & 13 & $\mathrm{NC}$ \\
\hline Tyrannus melancholicus & $\mathrm{R}$ & & & & 3 & & & 3 & & & 5 & 11 & $\mathrm{R}$ \\
\hline Tyrannus crassirostris & $\mathrm{R}$ & & & & & & & & & & 2 & 2 & $\mathrm{R}$ \\
\hline \multicolumn{14}{|l|}{ VIREONIDAE } \\
\hline Vireo pallens * & $\mathrm{R}$ & $\operatorname{Pr}$ & 4 & 1 & & 3 & & & & & 5 & 12 & $\mathrm{R}$ \\
\hline Vireo bellii * & M & & & & 1 & 3 & 1 & & 5 & 2 & & 9 & $\mathrm{R}$ \\
\hline \multicolumn{14}{|l|}{ CORVIDAE } \\
\hline Calocitta formosa * & $\mathrm{R}$ & & 2 & & & & & 9 & & & 12 & 23 & $\mathrm{NC}$ \\
\hline \multicolumn{14}{|l|}{ HIRUNDINIDAE } \\
\hline Tachycineta albilinea & $\mathrm{R}$ & & & & 5 & & & & & & 7 & 12 & $\mathrm{R}$ \\
\hline $\begin{array}{l}\text { TROGLODYTIDAE } \\
\text { Campylorhynchus }\end{array}$ & & & & & & & & & & & & & \\
\hline rufinucha * & $\mathrm{R}$ & & 25 & 5 & & 12 & 6 & & 10 & 4 & & 47 & $\mathrm{MC}$ \\
\hline $\begin{array}{l}\text { Thryothorus pleurostictus } \\
*\end{array}$ & $\mathrm{R}$ & & 9 & 4 & & 6 & 4 & & 12 & 6 & & 27 & $\mathrm{NC}$ \\
\hline Thryothorus felix * & $\mathrm{R}$ & & & & & & & & 4 & 1 & & 4 & $\mathrm{R}$ \\
\hline \multicolumn{14}{|l|}{ SYLVIIDAE } \\
\hline Polioptila caerulea * & M & & 4 & & & 5 & 1 & & 2 & & & 11 & $\mathrm{R}$ \\
\hline Polioptila albiloris & $\mathrm{R}$ & & & & & & & 2 & & & 2 & 4 & $\mathrm{R}$ \\
\hline \multicolumn{14}{|l|}{ TURDIDAE } \\
\hline Catharus ustulatus * & M & & & & & & 1 & & & & & 1 & $\mathrm{R}$ \\
\hline Turdus rufopalliatus * & $\mathrm{R}$ & & & & 2 & 7 & 4 & & 3 & 2 & & 12 & $\mathrm{R}$ \\
\hline \multicolumn{14}{|l|}{ PARULIDAE } \\
\hline Vermivora peregrina $*$ & M & & & & & & 1 & & & & & 1 & $\mathrm{R}$ \\
\hline Vermivora celata $*$ & M & & & & & 1 & 1 & & & & & 1 & $\mathrm{R}$ \\
\hline Parula americana * & M & & 5 & 3 & & 2 & 1 & & & & & 7 & $\mathrm{R}$ \\
\hline Dendroica petechia * & M & & 12 & 12 & & 21 & 17 & & 5 & 2 & & 38 & $\mathrm{MC}$ \\
\hline Dendroica magnolia * & M & & & & & & & & & 1 & & 1 & $\mathrm{R}$ \\
\hline Dendroica coronata $*$ & M & & & & & 2 & 4 & & & & & 2 & $\mathrm{R}$ \\
\hline Dendroica dominica * & M & & & & & & & & & 1 & & 1 & $\mathrm{R}$ \\
\hline Mniotilta varia $*$ & M & & 14 & 10 & & 15 & & & 8 & 2 & & 37 & $\mathrm{MC}$ \\
\hline Setophaga ruticilla $*$ & M & & 39 & 30 & & 42 & 27 & & 9 & 4 & & 90 & $\mathrm{C}$ \\
\hline Protonotaria citrea * & $\mathrm{M}$ & & & & & & & & 1 & 1 & & 1 & $\mathrm{R}$ \\
\hline
\end{tabular}


Apéndice 1. Continúa.

\begin{tabular}{|c|c|c|c|c|c|c|c|c|c|c|c|c|c|}
\hline \multirow[t]{2}{*}{ Orden, familia y especie } & \multirow[t]{2}{*}{ Estacionalidad } & \multirow[t]{2}{*}{$\begin{array}{c}\text { NOM- } \\
059\end{array}$} & & CHACAHUA & $H U A$ & \multicolumn{3}{|c|}{ MANIALTEPEC } & \multicolumn{3}{|c|}{ VENTANILLA } & \multirow[t]{2}{*}{$\begin{array}{l}\text { Total de } \\
\text { individuos } \\
\text { registrados }\end{array}$} & \multirow[t]{2}{*}{$\begin{array}{c}\text { Abundancia } \\
\text { relativa }\end{array}$} \\
\hline & & & In & $C p$ & $E x$ & In & $C p$ & $E x$ & In & $C p$ & $E x$ & & \\
\hline Seiurus aurocapilla * & M & & & & & 6 & 3 & & & & & 6 & $\mathrm{R}$ \\
\hline Parkesia noveboracensis * & M & & 69 & 60 & & 43 & 26 & & 13 & 2 & & 120 & A \\
\hline Oporornis tolmiei * & M & A & 2 & 1 & & 3 & 2 & & 1 & & & 6 & $\mathrm{R}$ \\
\hline Wilsonia pusilla * & M & & 2 & 2 & & & & 2 & & & & 4 & $\mathrm{R}$ \\
\hline Icteria virens $*$ & M & & 4 & 3 & & & & & & & & 4 & $\mathrm{R}$ \\
\hline \multicolumn{14}{|l|}{ THRAUPIDAE } \\
\hline Piranga rubra* & M & & & & & & 1 & & & & & 1 & $\mathrm{R}$ \\
\hline \multicolumn{14}{|l|}{ EMBERIZIDAE } \\
\hline Arremonops rufivirgatus * & $\mathrm{R}$ & & 2 & 1 & & & & 4 & 2 & 1 & & 8 & $\mathrm{R}$ \\
\hline \multicolumn{14}{|l|}{ CARDINALIDAE } \\
\hline Cardinalis cardinalis & $\mathrm{R}$ & & & & & & & & & & 6 & 6 & $\mathrm{R}$ \\
\hline Passerina cyanea * & M & & & & & & 1 & & & & & 1 & $\mathrm{R}$ \\
\hline Passerina leclancherii * & $\mathrm{R}$ & & & & & & & & 8 & 5 & & 8 & $\mathrm{R}$ \\
\hline $\begin{array}{l}\text { Passerina ciris * } \\
\text { ICTERIDAE }\end{array}$ & M & & 4 & 3 & & & & & 5 & 2 & & 9 & $\mathrm{R}$ \\
\hline Dives dives & $\mathrm{R}$ & & & & & & & & & & 8 & 8 & $\mathrm{R}$ \\
\hline Quiscalus mexicanus & $\mathrm{R}$ & & & & 5 & & & 3 & & & 22 & 30 & $\mathrm{NC}$ \\
\hline Icterus spurius * & M & & 3 & & & 3 & & & 7 & & & 13 & $\mathrm{NC}$ \\
\hline Icterus pustulatus * & $\mathrm{R}$ & & 3 & 1 & & 1 & & & & & 3 & 7 & $\mathrm{R}$ \\
\hline Icterus pectoralis * & $\mathrm{R}$ & & & & 2 & & & & 1 & & & 3 & $\mathrm{R}$ \\
\hline Icterus gularis * & $\mathrm{R}$ & & 2 & & & 5 & & & 6 & 2 & & 13 & $\mathrm{NC}$ \\
\hline Cacicus melanicterus * & $\mathrm{R}$ & & 10 & & & 17 & & & 8 & & & 35 & $\mathrm{NC}$ \\
\hline \multicolumn{14}{|l|}{ FRINGILLIDAE } \\
\hline Euphonia affinis * & $\mathrm{R}$ & & & & & & & & 2 & & & 2 & $\mathrm{R}$ \\
\hline
\end{tabular}


